\title{
ПРИНЦИПЫ ОРГАНИЗАЦИИ ФОРМАЛЬНО-КОГНИТИВНЫХ МОДЕЛЕЙ 1
}

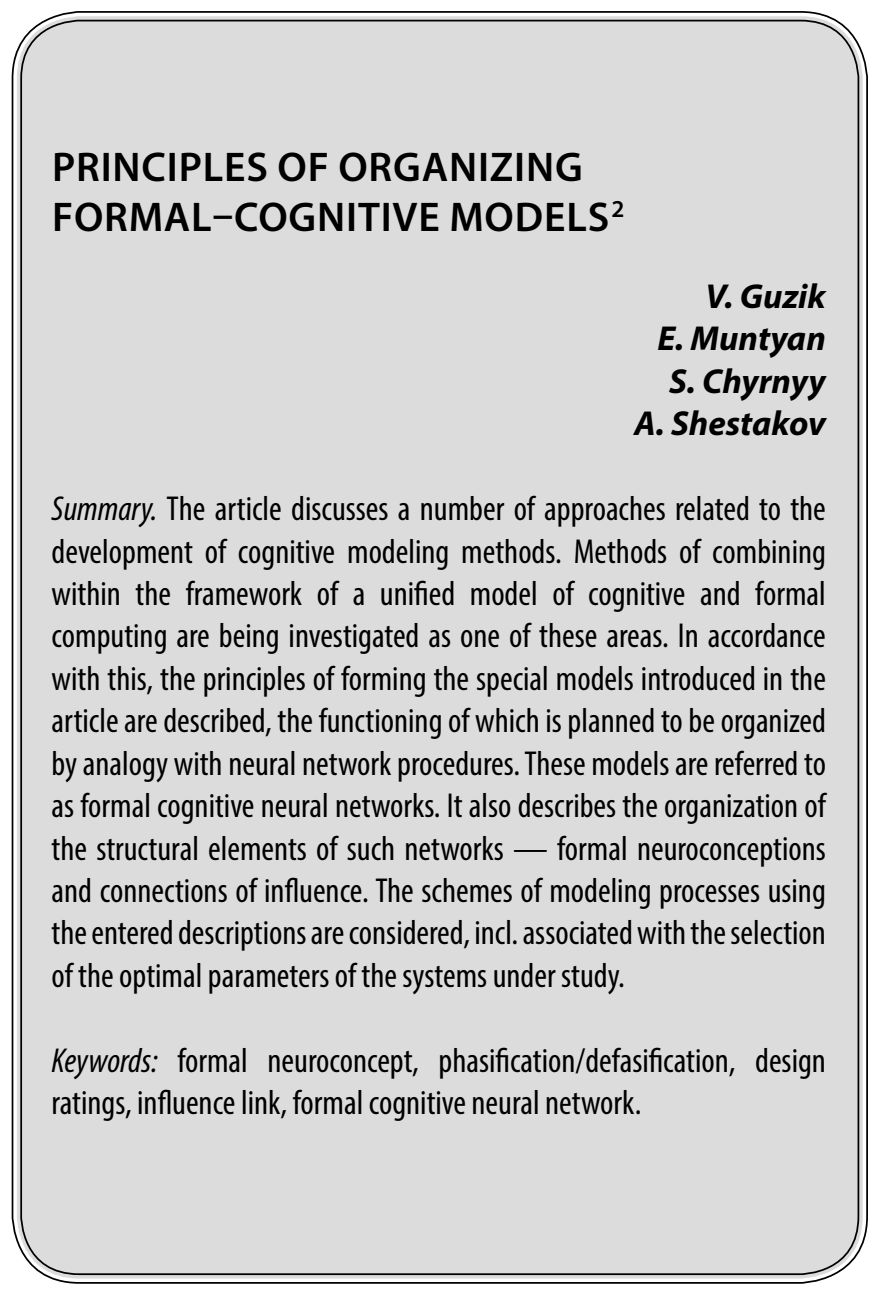

\section{Ввемение}

$\mathbf{0}$ дним из наиболее эффективных средств исследования слабоформализованных систем являются методы когнитивного моделирования $[1,2]$. Данные методы характеризуются достаточно серьезным спектром применений от атомной энергетики до транзитивной торговли $[3,4]$. Как известно, информационной основой данных методов являются субъективные оценки, формируемые специалистами-экспертами. Подобные оценки можно определить как виртуальное описание системы. Вместе с тем, для многих исследуемых объектов и процессов, которые будут в дальнейшем
Гузик Вячеслав Филиппович

Д.т.н., профессор, Южный федеральный университет

vfguzik@sfedu.ru

Мунтян Евгения Ростиславна

Дочент, Южный федеральный университет ermuntyan@sfedu.ru

Черный Сергей Александрович

К.т.н., дочент, Южный федеральный университет chernii.ser@yandex.ru

Шестаков Александр Валентинович

К.т.н., С.н.С., Южный федеральный университет trtualval@rambler.ru

Аннотация. В статье рассматривается ряд подходов, связанных с развитием методов когнитивного моделирования. В качестве одного из подобных направлений исследуются методы совмещения в рамках единой модели когнитивных и формальных вычислений. В соответствии с этим описываются принципы формирования вводимых в статье специальных моделей, функционирование которых планируется организовывать по аналогии с нейросетевыми процедурами. Указанные модели именуются, как формально-когнитивные нейросети. Описываются также организация конструкционных элементов подобных сетей - формальных нейроконцептов и связей влияния. Рассмотрены схемы процессов моделирования с использованием введенных описаний, в т.ч. связанные с подбором оптимальных параметров исследуемых систем.

Ключевые слова: формальный нейроконцепт; фазификация/дефазификация; проектно-рейтинговые оценки; связь влияния; формально-когнитивная нейросеть.

именоваться как исследуемые системы (ИС), имеется возможность, по крайней мере, частичной формальной оценки отдельных факторов, описываемых концептами когнитивных структур. Концепты, соответствующие подобным факторам, будут определяться как «гибридные концепты». Учитывая определенную общность топологической организации когнитивных сетей и нейросетевых моделей, в предлагаемой статье рассматриваются способы использования нейросетевых механизмов в процессах когнитивного моделирования.

Модели, совмещающие когнитивные (экспертные, расплывчатые) и реальные (формальные, четкие)

' Исследование выполнено при финансовой поддержке РФФИ в рамках научного проекта № 19-01-00412 и № 19-07-00570.

2 The reported study was funded by RFBR according to the research projects no.19-01-00412 and no. 19-07-00570 
оценки факторов ИС, а также реализуемые на основе элементов нейросетевых описаний, определяются в статье, как «формальная нейрокогнитивная сеть» (ФНКС). С точки зрения функционально-топологической организации ФНКС можно представить как сеть взаимодействующих элементарных процессоров, информационное взаимодействие которых осуществляется через соответствующие коммуникационные каналы, т.е. указанные процессоры и каналы связи являются основными конструктивными элементами ФКНС. Процессоры сети далее по тексту будут именоваться как функциональные нейроконцепты («фнк») или ф-нейроконцепты. Множество ф-нейроконцептов (Мн_ФНК $=\left\{\left.\mathbf{M}_{-} \phi \mathrm{HK}_{i l}\right|_{i l=1 \ldots 1}\right\}$, где м_фнк - модель $\phi$-нейроконцепта, I1 - мощность множества $\phi$-нейроконцептов) может быть структурировано в виде совокупности попарно непересекающихся подмножеств, как показано в (1).

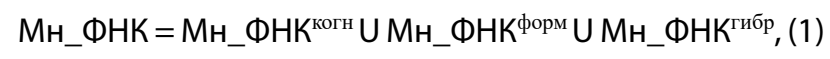

где $M_{H} \Phi H K^{\text {когн }}$ - подмножество когнитивных $\phi$-нейроконцептов, определяемых только когнитвной составляющей (формальная составляющая отсутствует);

$M H_{-} \Phi K^{\text {форм }}$ - подмножество формальных $\phi$-нейроконцептов, определяемых только формальной составляющей (когнитивная составляющая отсутствует);

$M H_{-} \Phi K^{\text {гибр }}$ - подмножество гибридных $\phi$-нейроконцептов, содержащих обе составляющие.

Каналы информационных коммуникаций между $\phi$-нейроконцептами будут именоваться «связями влияния» (св). Таким образом, ИС в процессе когнитивного моделирования представляется в виде ФНКС, причем факторы ИС соответствуют ф-нейроконцептам ФНКС, а причинно-следственные связи соответствуют связям влияния.

\section{МоАелИ констрУктИвных Э^ементов ФНКС}

Модель описания формального нейроконцепта описана в (2):

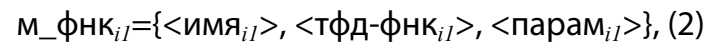

где $i 1=1 \ldots I 1-$ индекс/номер в сети описываемого $\phi$-нейроконцепта; I1 - число (мощность) множества;

$<$ имя $_{i l}>-$ текстовый идентификатор, обозначающий $\phi$-нейроконцепт;

$<$ тфд-фнк il $>$ - рейтинговая таблица фазификации/ дефазификации (будет рассмотрена ниже);

$<$ парам $_{i 1}>$ - значения основных параметров, характеризующих ф-нейроконцепт и определяющих его влияние на состояние сети;

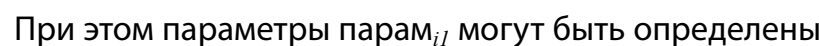
как:

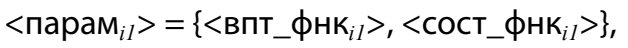

где <впт_фнк ${ }_{i 1}>$ - потенциал влияния на сеть $\phi$-нейроконцепта;

$<$ сост_фнк ${ }_{i 1}>-$ значение состояния $i 1$-го ф-нейроконцепта.

Следует отметить, что параметр сост_фнк ${ }_{i 1}$ соответствует по смыслу понятию состояния концепта в традиционных когнитивных моделях. Этот же параметр состояния концепта используется и как параметр, определяющий степень влияния концепта на сеть. В моделях ФНКС для внесения нелинейности вычислительного процесса вводится дополнительный параметр впт_фнк (потенциал выходного воздействия), который определяется, как функция от состояния ф-нейроконцепта и является параметром влияния на сеть. По аналогии с нейросетевым инструментарием в качестве функции предлагается использовать сигмоид [7], позволяющий обеспечивать усиление слабых сигналов и уменьшение насыщения от сильных.

Связи влияния в ФНКС обеспечивают информационное взаимодействие (коммуникации) между ф-нейроконцептами. Модель структуры св описывается в соответствии с (3).

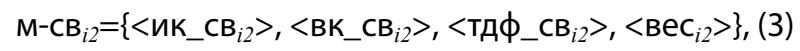

где $i 2=1 \ldots I 2-$ индекс/номер сети текущей $c в$;

I2 - число (мощность) множества моделей связей;

$<$ ик_св ${ }_{i 2}>$ - номер ф-нейроконцепта, являющегося исходящим для данной связи;

$<$ Вк_CB ${ }_{i 2}>-$ номер ф-нейроконцепта, в который данная связь направлена;

$<$ тдф_св ${ }_{i 2}>-$ таблица фазификации;

$<$ вес $_{i 2}>-$ вес связи, который можно рассматривать как коэффициент усиления значения выходного потенциала влияния ф-нейроконцепта, который является исходящим для рассматриваемой связи.

Связь влияния может быть ассоциирована с дугой графовой модели, причинно-следственной связью когнитивной модели и синаптической связью нейросетевой модели.

На рисунке 1 приводится функциональная структура гибридного ф-нейроконцепта, модели других типов могут быть представлены, как частные случаи приводимой модели.

Следует отметить основные особенности иллюстрируемой рисунком модели. 


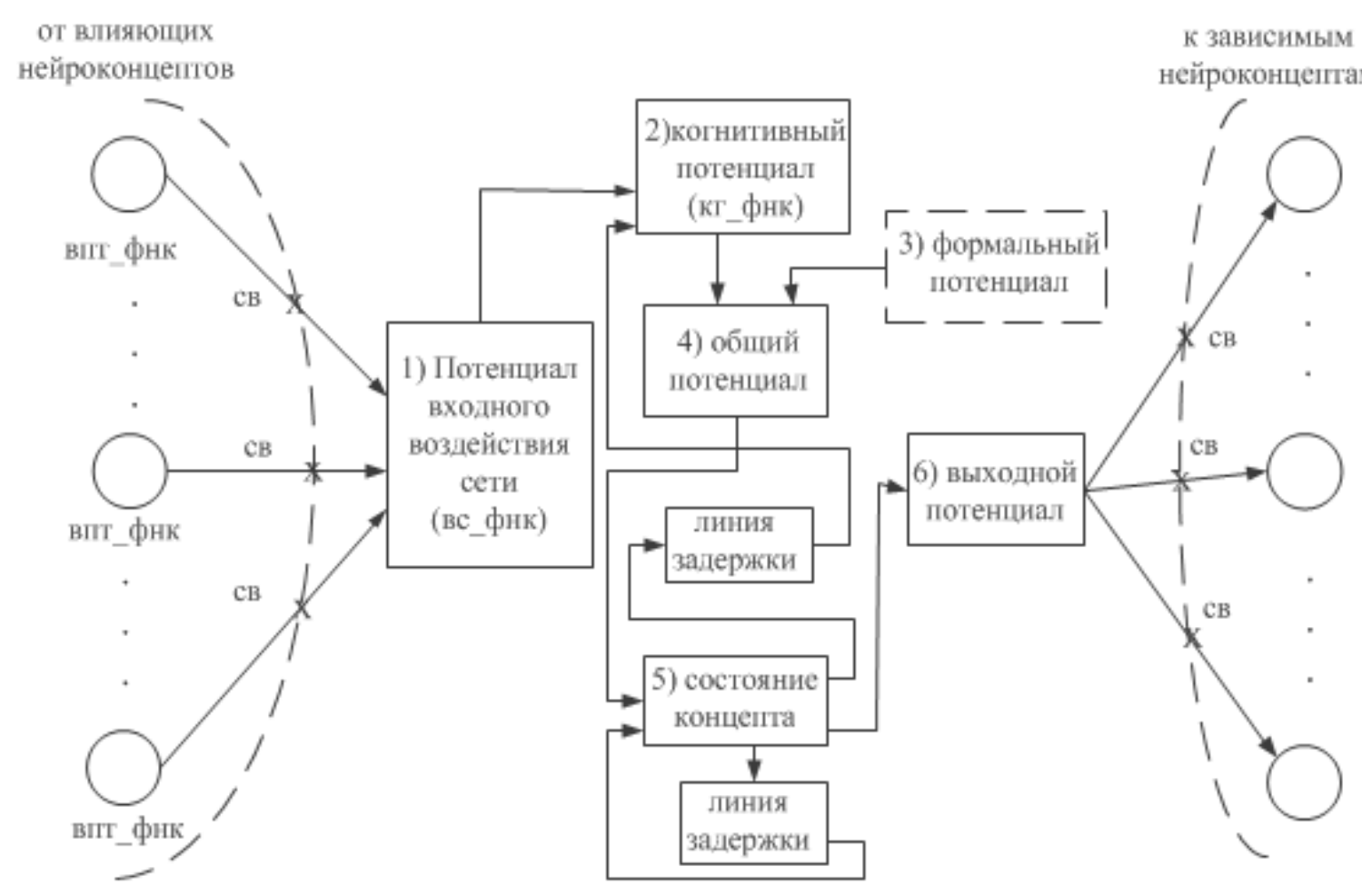

Рис. 1. Функциональная структура модели ФНКС

1) Модель ф-нейроконцептов в общем случае, как это рассмотрено выше, содержит две составляющие формальную и концептуальную. Однако в соответствии с предметной областью и прикладной спецификой формируемых моделей одна из составляющих может отсутствовать. Далее, как обобщающая, рассматривается гибридная модель.

2) Потенциал влияния входного воздействия сети (вс_фнк) определяет степень воздействия сети на состояния ф-нейроконцепта и определяется значениями состояний смежных потенциалов, усиленных соответствующими значениями (весами) связей влияния (блок1). Для описания механизма определения данного параметра вводится два функционала:

а) набор смежных ф-нейроконцептов входного воздействия по отношению к рассматриваемому ф-нейроконцепту $\mathrm{HBx}^{\mathrm{i}}$,

б) шаги моделирования по времени $-t_{k}$.

$\mathrm{HBX}^{\mathrm{i} 1}=\left\langle\mathrm{HBX}^{\mathrm{i} 1}{ }_{1} \ldots \mathrm{HBX}^{\mathrm{i} 1}{ }_{l} \ldots \mathrm{HBX}^{\mathrm{i} 1}{ }_{L}>\right.$.

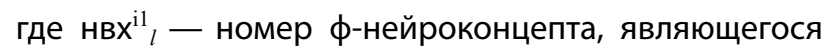
входным по отношению к рассматриваемому ( $i 1-$ му) $\phi$-нейроконцепту;

$L$ - общее число входных по отношению к рассматриваемому элементов.
BC_ $\phi \mathrm{HK}_{i l}\left(t_{k}\right)=\sum_{(l=1 \ldots L)}\left[\mathrm{B} \Pi \mathbf{\mathrm { B }} \_\phi \mathrm{HK}_{i l}\left(t_{k)} \cdot \mathrm{CB}_{l, i l}\left(t_{k)}\right]\right.\right.$.

3) Когнитивный потенциал, как характеристика когнитивной составляющей по аналогии с мембранным потенциалом в нейросетях [6] определяется рекурсивно (блок 2): $\left.\left(t_{k-1}\right)\right)$.

$\mathrm{K} \Gamma_{-} \phi \mathrm{HK}_{i l}\left(t_{k}\right)=f_{\kappa n}\left(\mathrm{~K} \Gamma_{-} \phi \mathrm{HK}_{i l}\left(t_{k-1}\right), \mathrm{BC} \_\phi \mathrm{HK}_{i l}\left(t_{k}\right)\right.$, сост_$\phi \mathrm{HK}_{i l}$

Здесь: $f_{\kappa n}$ - функция определения когнитивного потенциала.

4) Формальный потенциал определяется в соответствии с индивидуальными расчетными моделями, регламентирующими соответствующими методиками, связанными с конкретной ИС.

5) Общий потенциал ф-нейроконцепта (естественно, имеется в виду гибридный ф-нейроконцепт) определяется на основе двух составляющих — формальной и когнитивной:

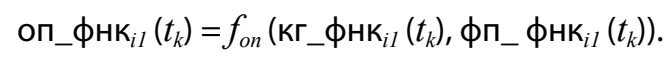

Здесь: $f_{\text {on }}$ - функция определения общего потенциала ф-нейроконцепта; 
фп_ фнк - формальный потенциал ф-нейроконцепта.

6) Состояние ф-нейроконцепта определяется следующим соотношением:

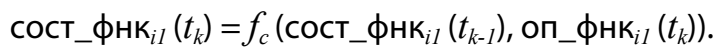

Здесь: $f_{c}$ - функция определения состояния.

7) Выходной потенциал ф-нейроконцепта определяет степень влияние ф-нейроконцепта на сеть. В традиционном представлении значение параметра состояния в когнитивных моделях используется и в качестве выходного воздействия на сеть. В предлагаемой схеме, по аналогии с моделями нейроэлементов, для формирования выходного воздействия предлагается введение нелинейности, например, сигмоида, что обеспечивает усиление слабых сигналов и компенсацию сильных. В этом случае выходной сигнал формируется как нелинейная функция от состояния ф-нейроконцепта (блок 6).

\section{Зак^ючение}

Введение формальной составляющей в процессы когнитивного моделирования позволяет определенным образом «приблизить» когнитивные, в достаточной степени имеющие субъективную (виртуальную) основу к реальной ситуации, подтверждаемой непосредственными измерениями или расчетными обоснованиями. Данное обстоятельство естественно обуславливает точность и объективность формируемых в ФНКС оценок в сравнении чисто когнитивным подходом.

К тому же формальная составляющая способствует повысить уровень адекватности когнитивной составляющей и детектирования необходимости и направления корректировки когнитивных представлений (как правило величины причинно-следственных связей, или, в терминах ФНКС - связей влияния). Мероприятия по корректировке моделей основывается на исследовании подмножества гибридных концептов, именуемых, как контрольные. Решения о необходимости корректировки принимается на основе нижеследующего соотношения $\varepsilon=\mid$ кг_фнк - фп_фнк $\mid \geq \Delta$, где $\varepsilon-$ невязка (расхождение) между формальной и когнитивной составляющих; $\Delta$ - порог допустимости, превышение которого сигнализирует о проблемах с корректностью модели.

Учитывая предлагаемый в статье нейросетевой подход к организации когнитивного моделирования в качестве образа нейроконцепта, используется специально разработанная модель динамического нейрона с памятью состояния [7]. Базой для реализации предложенных моделей и алгоритмов предполагается использование среда динамического моделирования технических систем SimlnTech [8].

\section{ЛИТЕРАТУРА}

1. Kolodenkova A.E., Muntyan E. R., Korobkin V.V. Modern approaches to modeling of risk situations during creation complex technical systems // Advances in Intelligent Systems and Computing. 2019. Vol. 875. pp. 209-217. D0l: 10.1007/978-3-030-01821-4_22.

2. Кулинич А. А. Когнитивная система поддержки принятия решений “Канва” / А.А Кулинич // Программные продукты и системы. - 2002. — № 3.— С. 2327.

3. Горелова Г. В. Когнитивный подход к имитационному моделированию сложных систем. Известия ЮФу. Технические науки. Издательство: Южный федеральный университет (Ростов-на-Дону 2013 № 3 239-250

4. Колоденкова А. Е. Построение системы когнитивных моделей и методов анализа реализуемости проекта по созданию информационно - управляющих систем для атомных станций / А. Е. Колоденкова // Глобальная ядерная безопасность — 2016. — № 3(20), С. 43-50.

5. Функции активации в нейронных сетях [Электронный ресурс].— URL: http://www.aiportal.ru/articles/neural-networks/activation-function.html

6. Чернухин Ю. В. Искусственный интеллект и нейрокомпьютеры. Таганрог: Изд-во ТРТУ. 1997, 273 с.

7. Guzik V. F. Fuzzy Cognitive Maps Considering Real Parameters of Simulated Systems / V. F. Guzik, A. S. Gamisonia, S. A. Chernyj, A. V. Shestakov // International Journal of Innovation, Creativity and Change. — 2019. — Volume 7, Issue - P. 153-160.

8. Карташов Б. А. Среда динамического моделирования технических систем SimlnTech / Б.А, Карташов, Е. А. Шабаев, 0. С. Козлов, А. М. Щекатуров - Москва: ДМК Пресс, 2017.- 423 с.

(с Гузик Вячеслав Филиппович ( vfguzik@sfedu.ru ), Мунтян Евгения Ростиславна ( ermuntyan@sfedu.ru ), Черный Сергей Александрович ( chernii.ser@yandex.ru ), Шестаков Александр Валентинович ( trtualval@rambler.ru ). Журнал «Современная наука: актуальные проблемы теории и практики» 